\title{
Fabry-Perot interferometer as a solar background noise suppressor: application to daytime lidar
}

\author{
Karnam Raghunath ${ }^{1, \star}$, Karnam Ramesh ${ }^{2}$, Sanama Narayana Reddy ${ }^{3}$ \\ ${ }^{1}$ National Atmospheric Research Laboratory, Department of Space, Gadanki, India \\ ${ }^{2}$ Sri Venkateswara University, Department of Physics, Tirupati, India \\ ${ }^{3}$ Sri Venkateswara University, Department of Electrical and Electronics Engineering, Tirupati, India
}

\author{
Article history \\ Received December 31, 2010; accepted September 12, 2011. \\ Subject classification: \\ Instruments and techniques, Lidar, Atmosphere, Daytime.
}

\begin{abstract}
Continuous atmospheric probing by a lidar is a requirement for many applications. However, due to high solar background noise during the daytime, lidar operations are mostly restricted to night-time. While many techniques are in practice, like reducing the receiver field of view, changing the view angle, introducing a narrow band Interference Filter (IF), these are applied to circumvent problems, rather than to suppress the noise. Using a Fabry-Perot interferometer as a narrow passband filter for solar background noise suppression is a known technique, and its potential is exploited in our system. An optical-fiber-coupled lidar system with its transmitter injection seeded was developed and has been operated during the daytime at Gadanki $\left(13.6^{\circ} \mathrm{N}, 79.2^{\circ} \mathrm{E}\right)$. The signal-to-noise ratio of the return signal is used as the performance indicator, to evaluate the improvements. Signal-to-noise ratios with and without the Fabry-Perot interferometer are measured with near identical test set-ups. The signal-to-noise ratio enhancement factor is ca. 4 , in agreement with the theoretical value. The performance is compared when the receiver fields of view are changed.
\end{abstract}

\section{Introduction}

The primary aim of this study is to demonstrate the feasibility of a lidar system for daytime measurements in the lower altitudes up to $30 \mathrm{~km}$, with the later extension of the profiling to the upper altitudes. Observations are limited to early morning and evening hours for vertically pointing lidars, without any solar background elimination methods. The extension of the lidar operation to daytime helps in the study of the properties of aerosols and clouds that might be affected in the presence of the sun. Thus lidar profiling of the atmosphere during the daytime is useful to compare the properties of various atmospheric constituents that can be changed in the presence of solar radiation. The signals received from the lidar during the daytime contain contributions from back-scattered signals at laser wavelengths, and unwanted noise signals due to solar radiation. The solar background noise is less during the early hours of the day, and becomes higher as the day progresses, and this noise strength needs to be suppressed to carry out meaningful observations.

Some of the main factors that contribute to the noise include the solar irradiance during the measurement time, the solar zenith angle, the wavelength used for profiling, the power of the laser, and the filters used. Some methods for reducing the background solar noise are as follows:

1. Making the telescope aperture size smaller; 2 . Using a wavelength that falls in the solar blind region; 3 . Reducing the field of view (FOV) of the receiver; 4. Changing the beam pointing angle according to the solar zenith angle; and 5. Reducing the optical system bandwidth using spectral filters in the receiver in conjunction with an injection seeded laser.

Three types of filters have been considered [Sidorin et al. 2005] in the past for use for such applications; namely, thin-film interference filters, birefringent filters, and etalonbased filters. Here it was concluded that etalon-based filters would be best-suited for cost-effective practical realization.

A pure rotational Raman lidar used a Fabry-Perot interferometer (FPI; M/s IC Optical Systems, UK) as a frequency comb filter to isolate return signals that are due to pure rotational Raman scattering from atmospheric nitrogen against the sky background [Arshinov et al. 2005]. Noctilucent clouds were observed during the daytime by appropriately equipping the lidar system with narrow optical filters in the form of FPIs [Blum and Fricke 2005], where a cut-off value was fixed to identify the clouds from the noise. A robust Rayleigh Mie lidar for daytime temperature profiling in the troposphere was developed simultaneously, using three techniques; namely, a narrow-band interference filter along with FPIs, the ultraviolet region of operation, and a narrow FOV to suppress the solar background noise [Hua et al. 2005], with good results. Using a FPI, a photometer was developed for the measurement of daytime OI 630.0-nm emission from the thermosphere [Narayanan et al. 1989]. In 


\begin{tabular}{ll}
\hline Transmitter & \\
Laser & Solid state class \\
Type & Nd:YAG, with seeding \\
Laser source & $532 \mathrm{~nm}$ \\
Operating wavelength & $600 \mathrm{~mJ}$ \\
Average energy per pulse & $7 \mathrm{~ns}$ \\
Pulse width & $50 \mathrm{~Hz}$ \\
Pulse repetition rate & $<0.1 \mathrm{mRad}($ after $10 \times$ expansion) \\
Beam divergence & $0.15 \mathrm{pm}$ with frequency stability of $50 \mathrm{MHz} / \mathrm{h}$ \\
Line width &
\end{tabular}

\section{Receiver}

Telescope

Type

Schmidt-Cassegrain

Diameter

$350 \mathrm{~mm}$

Field of view

Interference filter bandwidth

$0.4 \mathrm{mRad}$ with $F$ Number of 11

Maximum transmission

$1.07 \mathrm{~nm}$

Detector

$48 \%$

PMT, with gain of $34 \times 10^{7}$, dark current $50 \times 10^{-12} \mathrm{~A}$

Optical fiber

Type

Hard polymer clad, step index multimode fiber

Core diameter

$1.5 \mathrm{~mm}$ with numerical aperture of 0.37

Fabry-Perot interferometer

Type of the etalon

Material

Piezo tunable, capacitance stabilised air spaced etalon

Etalon clear (working) aperture

Schott Lithosil fused silica

Etalon FWHM

$50.8 \mathrm{~mm}$

Etalon cavity spacing

$2.08 \mathrm{pm}$ with reflective finesse of 7.2

$9 \mathrm{~mm}$

\section{Data Acquisition System}

PC based data acquisition system

operating with EG \& G ORTEC MCS software

Bin width (Range resolution)

$2 \mu$ s corresponding to $300 \mathrm{~m}$ with time integration of $250 \mathrm{~s}$

Table 1. Specifications of the lidar system.

micro-pulse lidar, the daytime background-induced noise is controlled by a narrow receiver FOV and a narrow bandwidth, temperature-controlled, interference filter [Spinhirne 1993].

There are cases where other techniques can be used as well. Daytime observation capability was realized with dualwavelength, high-altitude lidar sodium fluorescence channel by using a sodium atomic filter [Cheng et al. 2008, Hua et al. 2005]. A novel technique to reduce the sky background signal for a linearly polarized lidar was explored by using a polarization-selection technique [Hassebo et al. 2006] where the sky background noise is minimized by taking advantage of the naturally occurring polarization properties in scattered skylight. This has shown good improvements in the signal-to-noise ratio (SNR) over conventional schemes.

The proposed Raman lidar observations for daytime operations has a plan to use the third harmonic of a Nd:YAG laser, where the height coverage variations with different configurations have been discussed [Brunozzi and Marenco 2003] due to these schemes. An optimal design for suppressing solar scatter using single, double and triple etalons has been arrived at, and significant improvements have been achieved with single etalon itself. Subsequent addition of etalons is found to have incremental enhancement [McKay 1999]. Thus, daytime lidar observations were carried out with different techniques.

While the outcome is varied for these different methods, 


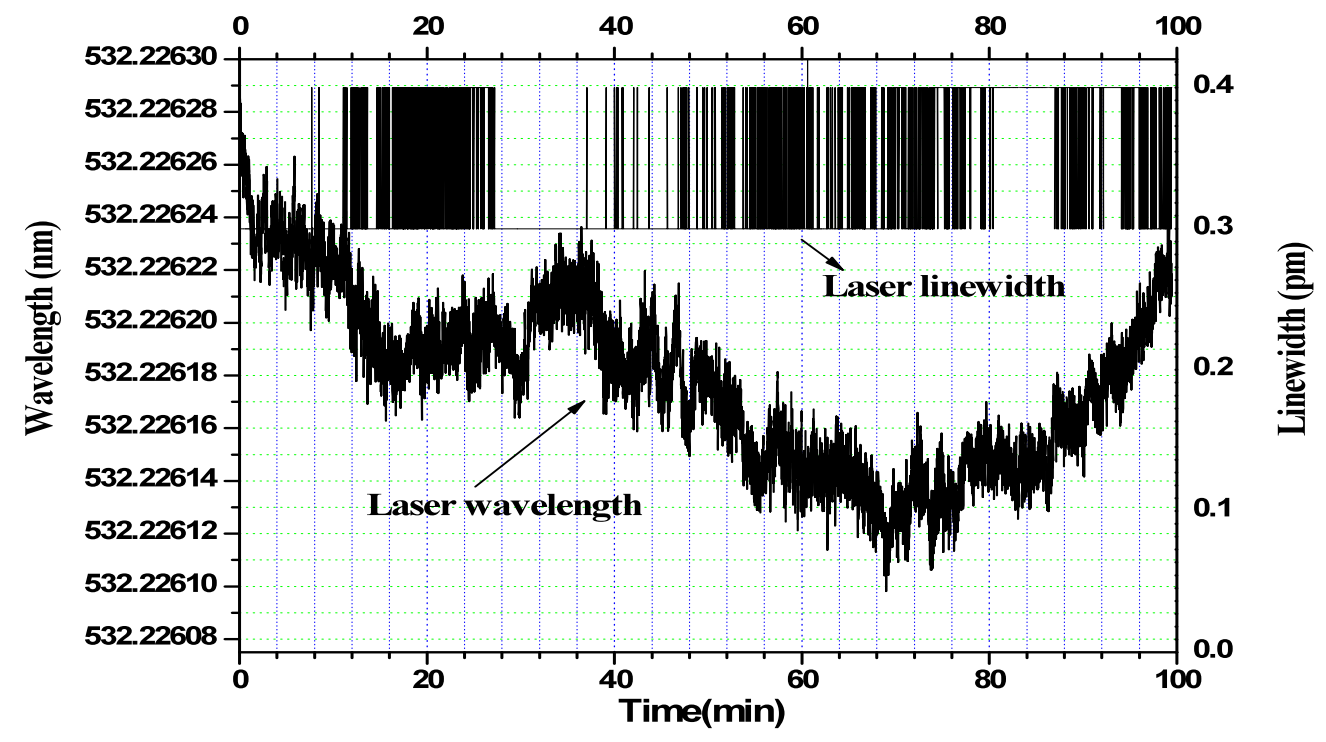

Figure 1. Frequency stability and line width of the laser, as measured with a wavelength meter.

the implementation depends on the cost effectiveness, the technology availability, the urgency, and the limitations and advantages. Here, in the present study we discuss the effects of using an etalon in the lidar receiver, and the improvements when compared with conventional methods.

\section{The lidar system}

The lidar system is a monotatic biaxial system with the laser output derived from a frequency doubled Nd:YAG laser. The laser beam is $8 \mathrm{~mm}$ in size, and the output power is 30 $\mathrm{W}$ at a pulse repetition frequency of $50 \mathrm{~Hz}$. The laser is injection seeded with a stable, narrow-line-width, continuous wave, fiber laser. The receiver is a $350-\mathrm{mm}$ Cassegrain feed telescope with an $F$ number of 11 , which is fiber coupled to the rest of the optics. The optical fiber has a numerical aperture of 0.37 , with core diameter of $1.5 \mathrm{~mm}$. The fiber diameter decides the FOV of the receiver. The photomultiplier tube (PMT) is used as a photodetector and has low dark noise, and it sends negative going pulses to a fast discriminator. After pulse shaping, the signals go to a multichannel scalar card for photon counting. The card functions in dwell and advance mode, and the photon strength is presented with respect to the lapse time. The functioning of an atmospheric lidar system is well known, and no further explanation will be given here. The specifications of the lidar system are given in Table 1, in a sufficient and elaborate manner.

The laser and etalon have a key role in the observations, and hence the characteristics are recorded. A model $\mathrm{n}^{\circ} \mathrm{WS}$ U-10 (M/s High Finesse) wavelength meter with absolute accuracy of $\pm 10 \mathrm{M} \mathrm{Hz}$ is used to measure the line width and the wavelength stability of the seeded laser (www.highfinesse. com). The laser characteristics are monitored for about $1.5 \mathrm{~h}$, and the results are shown in Figure 1. The line width consistently shows about $0.3 \mathrm{pm}$ on the right side of the plot. The wavelength drifts by ca. $0.1 \mathrm{pm} / \mathrm{h}$, which corresponds to ca. $300 \mathrm{MHz}$ and ca. $100 \mathrm{MHz} / \mathrm{h}$, respectively, in frequency a 532 $\mathrm{nm}$, as shown on the left side of the plot. The measurements are in agreement with those of the laser specifications.

The servo-stabilized FPI system comprises an etalon and a control unit. The control unit is a three-channel controller, which uses capacitance micrometers and piezoelectric (PZT) actuators to monitor and correct errors in mirror parallelism and spacing (www.icopticalsystems.com). The FPI is mounted on an XYZ mount for adjusting the angle of incidence of the optical fiber, to give additional control for the FPI. The Airy distribution of the etalon was obtained with a finesse of ca. 8.0 at $632.99 \mathrm{~nm}$, by scanning the etalon spacing for a few cycles with a stabilized helium-neon laser. The laser and etalon features are adequate for the present application. The general convention of terming the FPI as the etalon for fixed plate spacing is used throughout the text.

The SNR is one parameter that is used by many lidar researchers as a criterion to assess the lidar performance. The SNR is measured at the output of the photo detector by taking into account the total lidar instrument efficiency. The results of the performance of a lidar system that was operated during the daytime using an etalon is presented here. The etalon and fiber optics used in the present context are optimized for a direct detection lidar system, which is under development at the National Atmospheric Research Laboratory. Using the availability of the etalon, the lidar system is modified for daytime capabilities. The fiber optics need to be optimized for the $F$ number of the telescope, to obtain the designed height coverage [Jenness Jr. et al. 1997, Chourdakis et al. 2002]. For the present objective, where the etalon use as day-light filters is tested, this specification is not critical. Nevertheless, the performance of the lidar system that was expected is realized.

The working of the etalon for daytime applications has been explained for many studies [McKay 1999, Arshinov et al. 


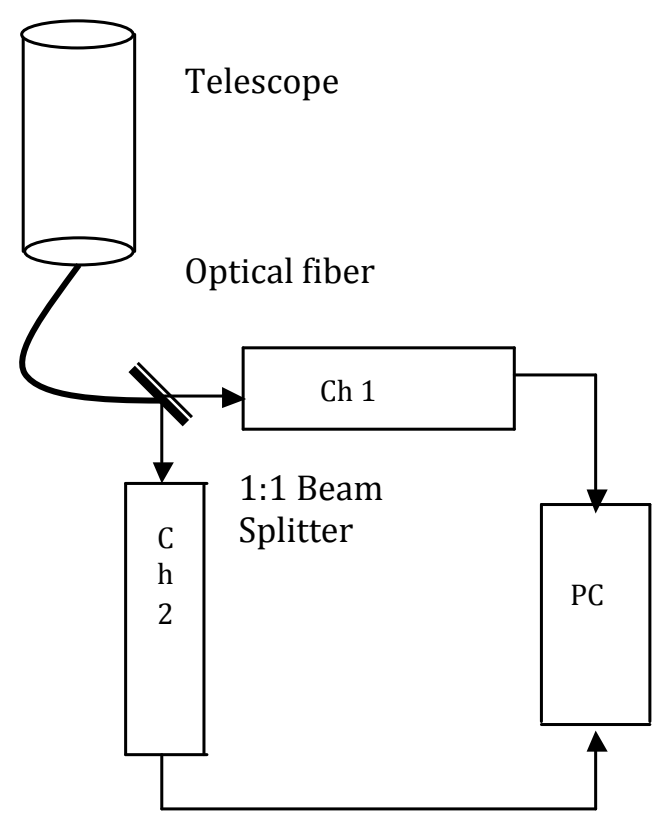

Figure 2. Receiver set-up of the lidar system used for testing the SNR improvement.

2005, Hua et al. 2005]. The SNR enhancement factor for the single etalon case is given by the approximate relation [McKay 1999] as:

$$
\mathrm{SNR}_{\text {Enhancement factor }}=2 / \pi^{\star} \mathrm{F}_{\mathrm{E}}
$$

where $\mathrm{F}_{\mathrm{E}}$ is effective finesse of the etalon.

Use of an etalon to isolate an individual spectral line of a lidar return against the continuum noise can be considered analogous to the isolation of narrow spectral intervals with interference filters, and the transmission enhancement is proportional to the number of peaks within a free spectral

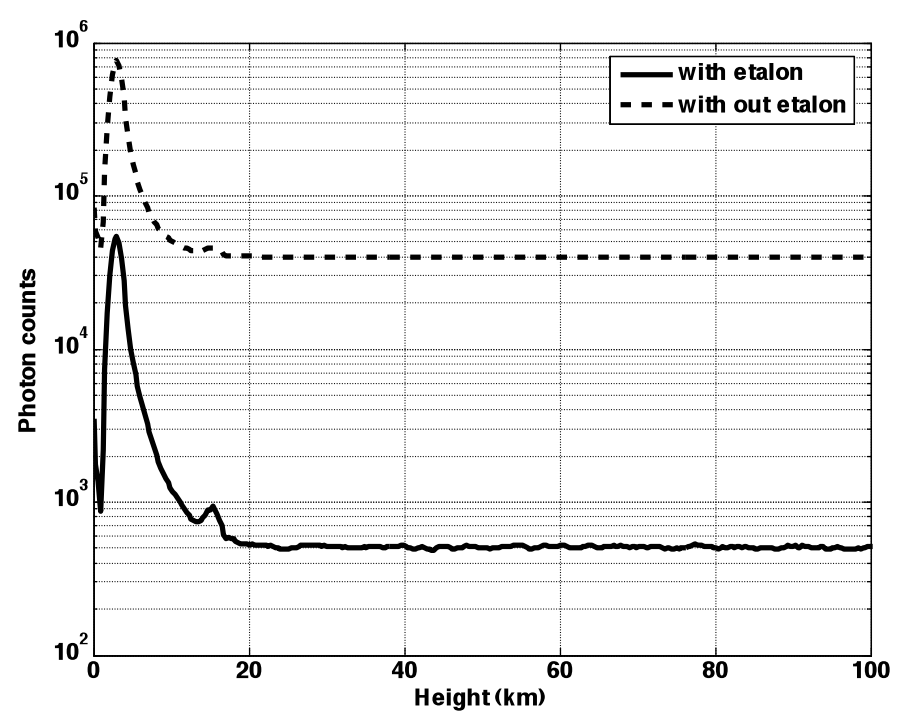

Figure 3. Raw data without the range correction, collected on March 4, 2010, at 0700 LT. The cloud presence is clearly visible in the 'with etalon channel'. range defined as the finesse [Arshinov et al. 2005]. This is the physical significance of the functioning of an etalon during the daytime. The SNR enhancement factor for a single etalon case cannot go beyond 10 usually, as high transmittance is needed for parameter extraction.

\section{The experiment}

To quantify the effects of using an etalon in the lidar receiver, an experiment was set up as shown in Figure 2. This has two channels in the receiver. The incoming beam is split into two equal signals with a 1:1 beam splitter, and it passes to one channel (Channel 1) that has etalon in the path, and another channel (Channel 2) that does not have an etalon. Apart from this difference, all of the other components, like the Interference Filter (IF), the lens assemblies, and the PMTs and their specifications, are identical for the two channels. This ensures near similar responses from the two receiving channels when no etalon is inserted, and hence in the actual experimentation, whatever signal changes that are observed between the Channels is due to the presence of the etalon only. The responses of the two channels are tested with a He:Ne laser. The difference between the two channels when no etalon is inserted was about $\pm 2 \%$, which can be ignored. To avoid saturation of the detector due to large returns from boundary layers, the fiber is slightly offset so that some of the signal is intentionally blocked.

\subsection{SNR estimation from the lidar data}

The lidar system was operated over several days with the above set-up. By comparing the SNR between two channels, the SNR enhancement/reduction factor of the signal is estimated. The SNR is calculated as given in Equation (2). The received signal is approximated to a Poisson distribution for detection:

$$
\operatorname{SNR}(r)=\frac{N(r)}{P_{b k g}+\sqrt{N(r)}}
$$

where $N(r)$ is the returns from the range $r$, and $P_{b k g}$ is the returns due to the solar background.

$P_{b k g}$ is estimated from the returns at the upper heights, typically at about $500 \mathrm{~km}$, where the 'wanted signal' is minimum and all of the contribution is due to solar noise only. The dark noise that is due to the PMT is common for both channels, and hence this is not considered. The solar zenith angle has a crucial role, and its value at Gadanki $\left(13.6^{\circ} \mathrm{N}, 79.2^{\circ} \mathrm{E}\right)$ is calculated from the information given on the website of the U.S. Naval Observatory Astronomical Applications Department (http:/ / aa.usno.navy.mil/ data/ docs/AltAz.php).

The term of a SNR enhancement/ reduction factor is introduced to quantify the SNR changes during the daytime/night-time. This is expressed as a ratio of the SNR between Channel 1 and Channel 2. 


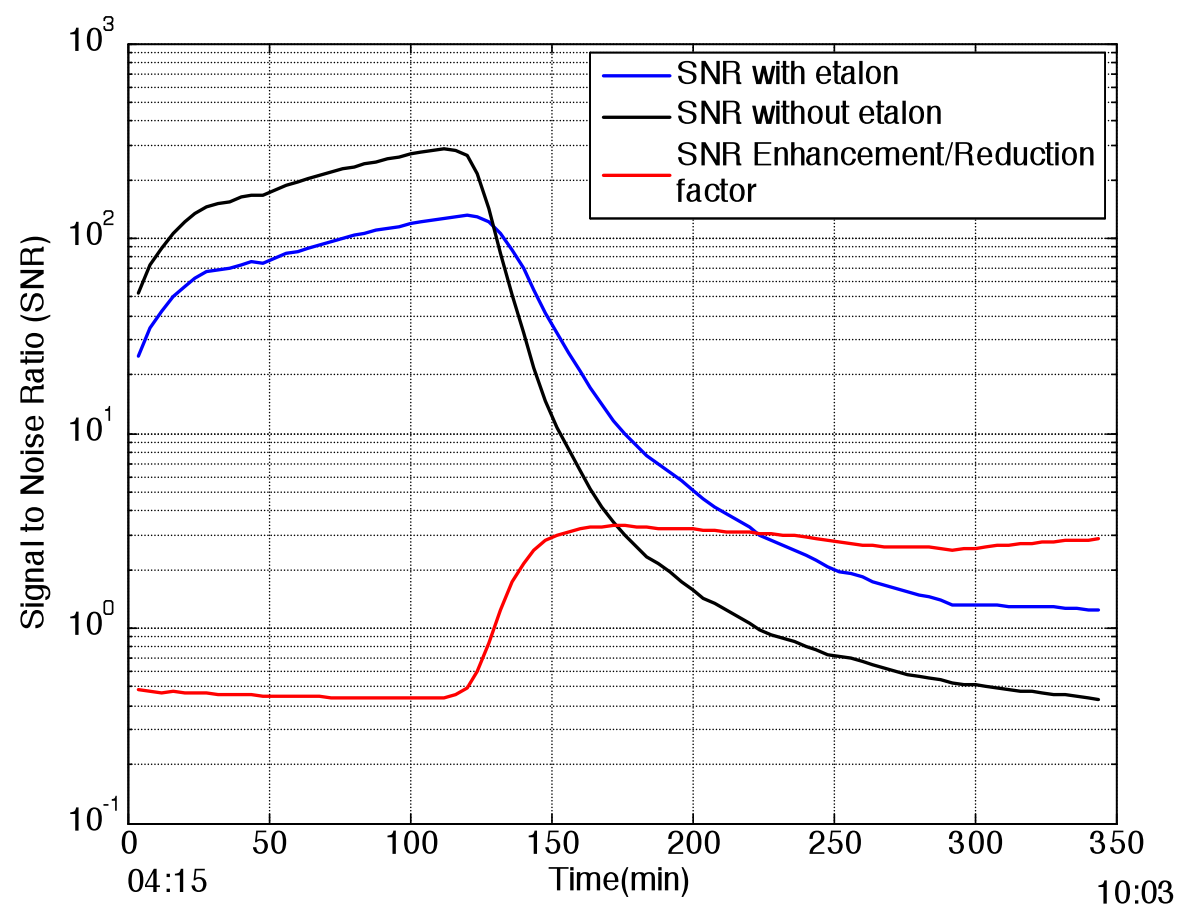

Figure 4. Lidar SNR values obtained at an altitude of $10 \mathrm{~km}$ on March 18, 2010. The times of the observations are given at the extreme ends of the plot, as local time. $\Psi$ is solar zenith angle.
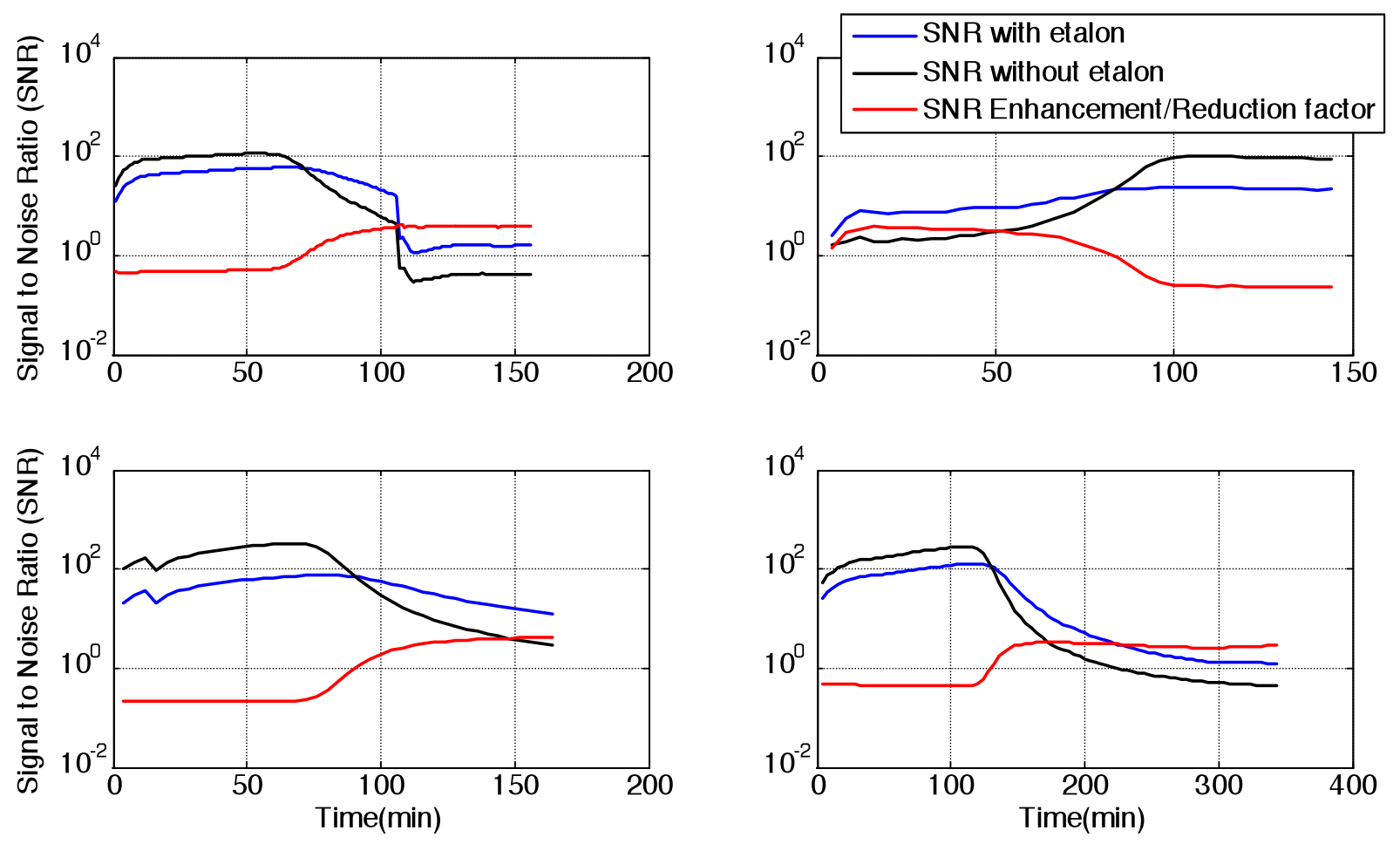

Figure 5. Lidar SNR and SNR enhancement/reduction factors obtained at an altitude of $10 \mathrm{~km}$ on different days. Observation times: (a) 0505 to $0740 \mathrm{LT}$; (b) 1708 to $1930 \mathrm{LT}$; (c) 0510 to $0754 \mathrm{LT}$; (d) 0415 to $1003 \mathrm{LT}$. Sun positions at pointer: (a) $0610 \mathrm{LT}, \mathrm{SZA}(\psi) 93^{\circ}$; (b) $1840 \mathrm{LT}, \mathrm{SZA}(\psi) 95^{\circ}$; (c) $0610 \mathrm{LT}, \mathrm{SZA}$ ( $\psi$ ) $92^{\circ}$; (d) 0615 LT, SZA $(\psi) 93^{\circ}$.

\section{Results and discussion}

\subsection{SNR improvement}

The raw photon count profiles from both of the channels for a day are shown in Figure 3, in which cloud presence can clearly be seen.
The SNR profile for the data collected without and with the etalon channels (Channel 1 and Channel 2 ) and SNR enhancement/reduction factor at the particular altitude of $10 \mathrm{~km}$ is shown in Figure 4, for March 18, 2010. The measurements were taken during 0415-1003 LT, which extends from early morning to late morning. The SNR factor 


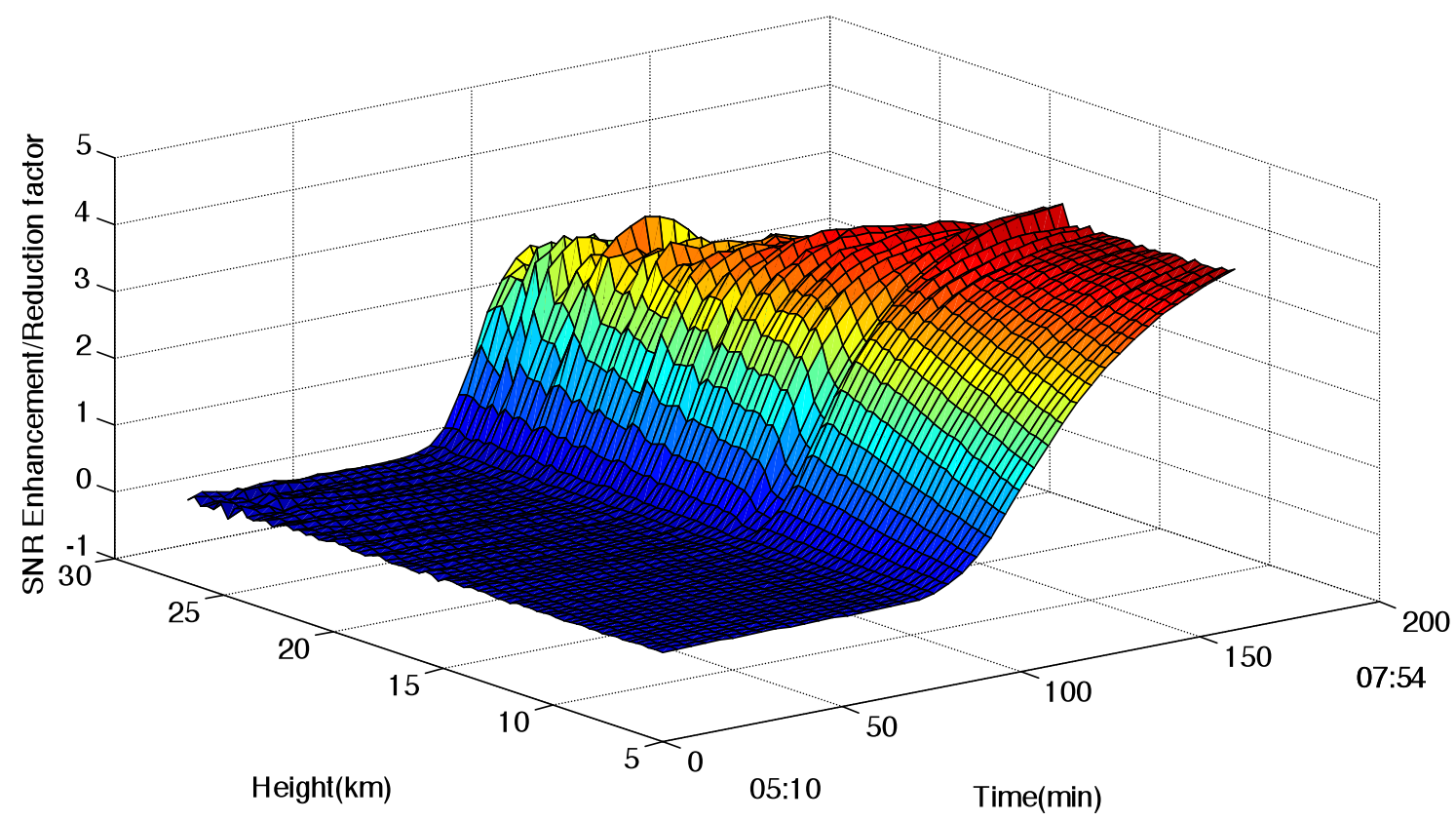

Figure 6. Plot showing the SNR enhancement/reduction factor for heights up to $25 \mathrm{~km}$ on March 4, 2010, from 0510 to $0754 \mathrm{LT}$. The times of the observations are given at the extreme ends of the plots, in local time. A near constant factor is seen for the full height coverage for the entire duration of the observation.

shows constant reduction during the night-time and it starts to be enhanced from day-break. This corresponds to a solar zenith angle $(\psi)$ of $93^{\circ}$ at $0615 \mathrm{LT}$, and of $37^{\circ}$ at $1003 \mathrm{LT}$.

Case 1: During the night-time (0415-0615 hours), when the solar noise is less or nil, Channel 1 attenuates more of the desired signal in the total signal (desired + noise) and less or none of the solar noise. Channel 2 does not provide any attenuation. This results in lower SNR values in Channel 1 and better SNR values in Channel 2. This explains less than one SNR reduction factor during night-time.

Case 2: During the daytime, Channel 1 (0615-1003 LT) attenuates the same amount of the desired signal in the total signal (desired + noise) as during the night-time, but it attenuates more of the noise in the total signal because of the high solar noise. As in the previous case, Channel 2 does not provide any attenuation. This results in lesser SNR values in Channel 2, and better SNR values in Channel 1. This explains the flat region of the SNR enhancement factor of about 4 during the daytime. This is in agreement with Equation (1), which gives the relationship between the SNR enhancement factor and the finesse of the etalon.

The results of this experiment carried out over 4 days is shown in Figure 5. While Figure panels a, c and d of Figure 5 shows observations from the night-time to the daytime, Figure $5 \mathrm{~b}$ shows observations from the daytime to the nighttime. As the room temperature rose above normal operating conditions, the lidar operations could not be carried out around midday. Some of the observations carried out during the pre-noon hours are expected to be valid during the postnoon hours, ignoring the effects of clouds, if any.
Nevertheless, the SNR enhancement factor shows the trend of maintaining ca. 4, as is evident in Figure 5. This is in agreement with the relationship between the finesse of the etalon and the SNR enhancement factor [McKay 1999]. The whole height coverage has a similar performance, as shown in Figure 6 for March 4, 2010. The constant SNR reduction factor during the post-sunset and pre-sunrise times is as expected. It should be noted that in spite of the variations in the individual SNRs (Channel 1 and Channel 2), the SNR enhancement/reduction factor is not affected.

\subsection{Comparison with the conventional method}

The effectiveness of using etalon as a noise suppressor was compared with the conventional method of reducing the receiver FOV. The experiment was conducted on March 18, 2010, alongside an identical receiver set-up with a FOV of $1 \mathrm{mRad}$. The results plotted in Figure 7 have a similar trend as seen when the etalon was used, although the effect is different. Though the SNR reduction factor is the same (ca. 0.45 ) for both cases, the SNR enhancement factor is ca. 1.5 only. It is clear that the etalon as a solar noise suppressor is more effective than the method of reducing the FOV, although this latter method is economical and easy to implement. It is expected that the characterization is valid for any FOV reduction of a factor of 0.4 .

\subsection{Height coverage improvements}

To determine the height coverage improvement with the noise suppression methods, the SNR profiles in Figure 8 were considered for the March 18 data at 0733 LT. This is summarized in Table 2, which implies that the height coverage improvement is better when the etalon is used in the 


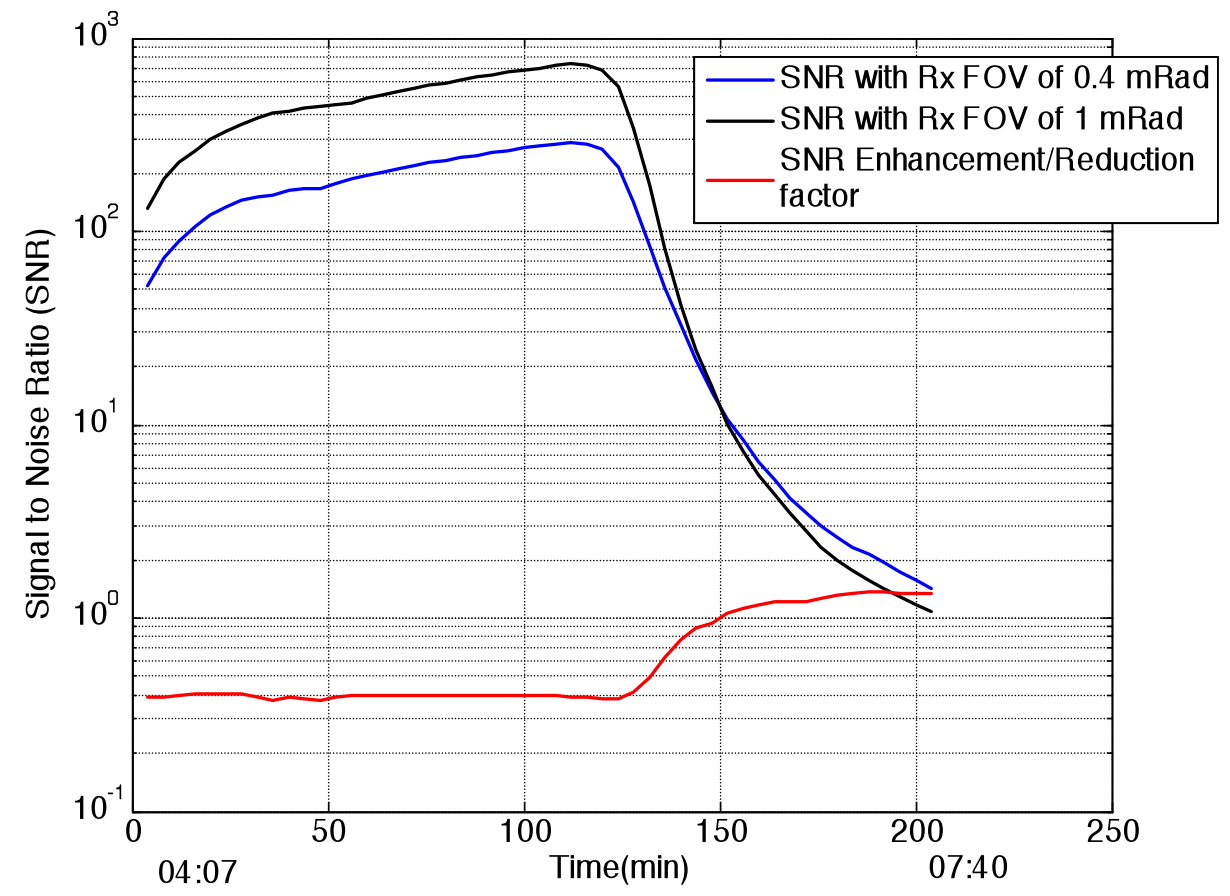

Figure 7. Lidar SNR values obtained at an altitude of $10 \mathrm{~km}$ on March 18, 2010, for a change in the FOV. The times of observations are given at the extreme ends of the plots, in local time.
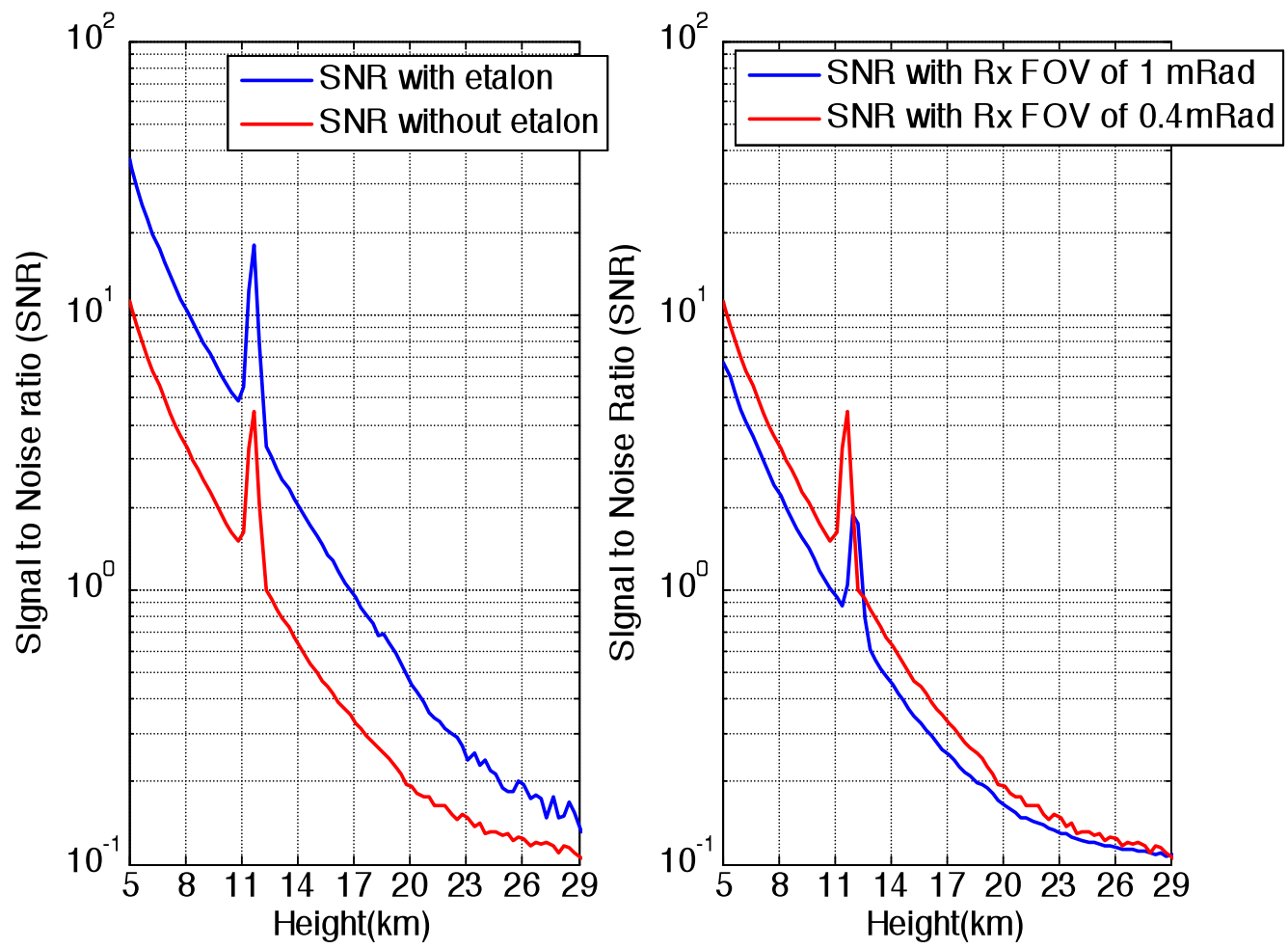

Figure 8. Height gain at 0733 hours on March 18, 2010, with the two techniques. The height coverage advantage is clear from the two techniques, where the SNR is one.

\begin{tabular}{ccccc}
\hline Method & Change in $\mathrm{SNR}_{\text {night time }}$ at $\mathbf{0 5 0 0}$ & Change in $\mathrm{SNR}_{\text {day time }}$ at 0730 & $\begin{array}{c}\text { Altitude coverage gain during } \\
\text { day time at } \mathbf{0 7 3 0}(\mathbf{a t} \mathbf{S N R}=\mathbf{1})\end{array}$ \\
\hline Using etalon as spectroscopic filter & Reduction by a factor of ca. 0.45 & Enhancement by a factor of ca. 4 & ca. $5 \mathrm{~km}$ \\
Reducing FOV of the receiver & Reduction by a factor of ca. 0.4 & Enhancement by a factor of ca. 1.5 & ca. $1 \mathrm{~km}$ \\
\hline
\end{tabular}

Table 2. Operational performance of two methods. 
receiver chain. The best performance from an etalon-based receiver can be achieved when its maximum finesse is used.

For most cases, $\mathrm{SNR}=1$ is the criterion for carrying out cloud and related studies. The height improvement of 33\% at $\mathrm{SNR}=1$, as shown in Figure 8 for the etalon-based receiver, can be compared with the achieved value of $34 \%$ by the polarization discrimination technique [Hassebo et al. 2006] at $S N R=10$. Further, SNR at the cloud top is good, to build a slope for retrieving the atmospheric parameters.

It is expected that using one or more methods for noise suppression will improve the overall performance.

\section{Conclusions}

The operation of a daytime lidar is demonstrated using etalons as the narrow passband filter for suppressing the solar background noise. The theoretical improvement in the SNR has been realized. The effect of the presence of the etalon during the night-time and daytime was studied, and its superior performance over the conventional method of the changing of the receiver FOV is noted. This technique can be used effectively for studies of diurnal variations of aerosol concentrations and cloud dynamics.

\section{References}

Arshinov, Y., S. Bobrovnikov, I. Serikov, A. Ansmann, U. Wandinger, D. Althausen, I. Mattis and D. Müller (2005). Daytime operation of a pure rotational Raman lidar by use of a Fabry-Perot interferometer, Appl. Optics, 44, 3593-3603.

Blum, U., and K.H. Fricke (2005). The Bonn University lidar at the Esrange: technical description and capabilities for atmospheric research, Annales Geophysicae, 23, 1645-1658.

Cheng, X., S. Gong, F. LiI, Y. Dai, J. Song, J. Wang and F. Li (2008). Daytime observation technology of a lidar using an atomic filter front, Frontiers of Optoelectronics in China, 1 (3-4), 274-278.

Chourdakis, G., A. Papayaannis and J. Porteneuve (2002). Analysis of the receiver response for a noncoaxial lidar system with fiber-optic output, Appl. Optics, 41, 27152723.

Hassebo, Y.Y., B. Gross, M. Oo, F. Moshary and S. Ahmed (2006). Polarization-discrimination technique to maximize the lidar signal-to-noise ratio for daylight operations, Appl. Optics, 45, 5521-5231.

Hua, D., M. Uchida and T. Kobayashi (2005). Ultraviolet Rayleigh-Mie lidar for daytime-temperature profiling of the troposphere, Appl. Optics, 44, 1315-1322.

Jenness Jr., J.R., D.B. Lysak and R. Philbrick (1997). Design of a lidar receiver with fiber-optic output, Appl. Optics, $36,4278-4284$.

McKay, J.A. (1999). Single and tandem Fabry-Perot etalons as solar background filters for lidar, Appl. Optics, 38, 5851-5858.
Narayanan, R., J.N. Desai, N.K. Modi, R. Raghavarao and R. Sridharan (1989). Dayglow photometry: a new approach, Appl. Optics, 28, 2138-2142.

Sidorin, Y., D. Lun and B.R. Chou (2005). Etalon-based filters for solar and atmospheric research, Opt. Eng., 44, 076001 (Jul 14, 2005); http:/ / dx.doi.org/10.1117/1.1947784.

Spinhirne, J.D. (1993). Micropulse lidar, IEEE T. Geosci. Remote, 31, 48-55.

Tini Brunozzi, P., and F. Marenco (2003). A lidar for water vapour measurements in daytime at Lampedusa, Italy, Annals of Geophysics, 46 (2), 459-465.

\footnotetext{
${ }^{\star}$ Corresponding author: Karnam Raghunath, National Atmospheric Research Laboratory, Department of Space, Gadanki, India; email: kraghunath@narl.gov.in.
}

(C) 2012 by the Istituto Nazionale di Geofisica e Vulcanologia. All rights reserved. 